\title{
Multicultural competencies dealing with people who have sleep disorders
}

\begin{abstract}
With the different types of sleep disorders apparent to many allied health professionals including medical doctors, the need to understand the diversity of patient symptoms is important. For example, sleep has resulted in many being fired from their jobs because employers do not understand the condition. Having said that, understanding the diversity of treatment professionals might be more important because of the various worldviews depending on what part of the world we are from. Thus, patients may receive counterproductive treatment depending on the culture (e.g., race, ethnicity, tribe, religion) the patient is from. This brief article deals with understanding what multicultural competencies are needed to treat every patient with respect, equality, and dignity while treating their sleep disorder.
\end{abstract}

Keywords: sleep disorders, multiculturalism, diversity, application, multicultural counseling competencies
Volume 2 Issue I - 2018

\author{
Keith BWilson,' Carrie L Acklin, ${ }^{2}$ Si-Yi \\ $\mathrm{Chao}^{3}$ \\ 'Department of the Rehabilitation, Southern Illinois University \\ Carbondale, USA \\ ${ }^{2}$ Department of Rehabilitation and Counseling, University of \\ Wisconsin-Stout, USA \\ ${ }^{3}$ Doctoral student in the Rehabilitation, Southern Illinois \\ University Carbondale, USA
}

Correspondence: Keith BWilson, Department of the

Rehabilitation Institute, Southern Illinois University Carbondale, Rehabilitation Institute, Rehn Hall, RM. 339A-Mail Code 4609, IL 6290I, USA, Email kbwilson@siu.edu

Received: December 13, 2017 | Published: February 15, 2018

\section{Introduction}

Unfortunately, patients can be discriminated against because of many personal attributes (e.g., race, ethnicity, tribe, religion) that may cause substandard care in treating sleep and other disorders. ${ }^{1}$ People with sleep disorders are no exception. For example, the World Health Organization (WHO, 2017) ${ }^{2}$ reported that depression is associated with several related symptoms including but not limited to anxiety, appetite and sleeping. ${ }^{2}$ WHO also reported that approximately 300 million people are living with depression and a host of other conditions that are positively correlated with sleep concerns? More specifically, WHO noted that mental health conditions are also associated with difficulty in sleeping? Speaking of mental health, several underrepresented groups tend to surfer more from mental health concerns than other groups. ${ }^{3}$ Thus, it stands to reason that generalizing from prior research looking underrepresented groups and discrimination, ${ }^{4,5}$ people who are part of underrepresented groups are likely to have sleeping concerns if they represented proportionally high with behavioral health concerns. ${ }^{6,7}$ While there are several variables that are associated with sleep conditions, depression and other related behavioral health condition are positively correlated with sleep disturbances, as well as being a person who is part of an underrepresented group.

When looking at the United States, for example, groups that might not be domestic encounter many gaps in receiving the needed services they are seeking, as well. For example, when seeking behavioral health services, Blacks are more and likely to have a low utilization rate when compared to Whites. O'Hara ${ }^{3}$ recently noted that in the UK, high levels of racial discrimination, for example, are reported by people who have a mixture of Indian, African, and Bangladeshi. When looking at the utilization of health care and psychological services in the United States, people who classify as Latino tend to take advantage of using these services more than Blacks, but less than Whites. Last, when we look at those with a psychiatric disorder who use behavioral health services in the United States, groups who are immigrants (e.g., African, Asian, Caribbean) tend to use these services far less than individuals who are domestic (Asian Americans, African Americans. ${ }^{8}$ While there are many reasons why groups tend to receive different frequencies of services and reported higher levels of discrimination in the United States and the UK one cannot discount the lack of cultural competency of the professionals in providing the same level of services to all of their patients.

\section{Bias service providers}

In the United States and the UK, groups who are not European American (e.g., Caribbean) tend to encounter more discrimination when interacting with many agencies and services providers. To highlight this observation, the Substance Abuse and Mental Health Services Administration (SAMHSA, 2015) ${ }^{9}$ noted the higher number of African Americans being hospitalized compared to European Americans (1.4\% \& $0.7 \%$, respectively) for inpatient mental health concerns. Another study underscored the likelihood that race and ethnicity tend to predict a diagnosis independent of symptoms. ${ }^{9}$ Although many training programs prepare their students to hopefully give all populations equitable service delivery, the application of such concepts tends to decrease in actual practice for many. What follows is a brief description of competencies to facilitate services for all patients entering our doors for help. These competencies are taking from the Multicultural Counseling Competencies produced by Sue, et al. ${ }^{10}$ 


\section{Needed competencies to decrease counterproductive outcomes}

Awareness:attitudes and beliefs: To modify counterproductive behavior, one must be cognizant of the behavior that might be yielding negative outcomes from certain groups we serve. ${ }^{10}$ Thus, being aware of negative views held towards certain group will be the first step to changing those views and behaviors. Further, healthcare professionals must be aware of the unique needs of each patient and how the patient's needs might differ when dealing with members of underrepresented groups. For example, it is well established in the literature that women's symptoms tend to be attributed to a mental health disorder rather than a medical disorder. ${ }^{7}$ What this might look like in practice is a physician attributing the woman's lack of, or interrupted sleeping patterns, to anxiety rather than a medical condition, such as sleep apnea. This is only one of several examples of where lack of awareness can lead to ineffective medical treatment.

Knowledge: Once aware of counterproductive thoughts and behaviors towards certain groups of people, it is important to gather information to help understand how to change these negative thoughts. ${ }^{10}$ Gaining knowledge can be as simple as educating oneself on cultures and traditions of the clientele being services by your hospital, for example. Healthcare professionals can also gain knowledge by asking the patient questions that go beyond the medical assessment so that the professional can gain additional knowledge about the unique needs of the patient. For example, asking the patient how their demographic variables (e.g., sex, disability status, gender, religion and tribe) might contribute to what the patient is experiencing, if at all.

Skills: Once you have the awareness and knowledge, skills are the tools you can put into action and apply what you have learned in changing counterproductive behaviors that clinicians might be projecting onto their patients..$^{10}$ It is imperative that the healthcare professional is implementing the skills in a meaningful way. The healthcare professional can check in with patient to make sure that the patient's symptoms and medical information are being understood in the right context, or the healthcare professional can read the patient's body language to assess whether or not the skills and tools that are being used are being used in the proper manner. For example, if it is noticed that the patient was open during the first part of the appointment, but became quiet and reserved as the appointment progressed, it might be the case that the skills and tools that were being used were not being used properly.

\section{Conclusion}

Because of the many types of sleep disorders and the diverse nature of the patients who use these services as well as the healthcare professionals who service this population, it is important to understand not only the disorder, but the multicultural nature of the patient. Research suggests that people who are different (e.g., race, ethnicity, tribe, religion) in the United States tend to be treated in counterproductive ways by health care professionals. Because of the enculturation process, most of us have certain thoughts about groups that might not be effective in facilitating fair and unbiased services in a healthcare setting. First, we must be willing to change our counterproductive behavior towards groups we may not view in a positive way. Next, we need to be able to continuously acquire knowledge related to patients and their unique and diverse needs and how these needs are not universal across all populations. Last, we must be able to develop and implement culturally competent skills in a healthcare setting. The interconnection between culturally competent awareness, knowledge, and skills can help facilitate effective, fair, and equal treatment for patients of all types.

\section{Acknowledgements}

None.

\section{Conflicts of interest}

The author declares no conflict of interest.

\section{References}

1. Evans TS, Berkman N, Brown C, et al. Disparities within serious mental illness. Agency for Healthcare Research and Quality (US), Technical Briefs. 2006. 25 p.

2. World Health Organization. "Depression: Let's talk" says WHO, as depression tops list of causes of ill health. Geneva: WHO; 2017.

3. O'Hara M. Mental health and race-the blight of dual discrimination. UK; 2014.

4. Wilson KB, Pitt JS, Raheem MA, et al. Multicultural counseling competencies: Why is it difficult to apply what we know...? In: Leavitt L, et al. editors. Cultural Awareness and Competency Development in Higher Education. IGI Global, Pennsylvania, USA: Hershey; 2017. p. 237-254.

5. Wilson KB. What does it mean to be a culturally-competent counselor? Paper presented at the meeting of the American Counseling Association. Multicultural Social Justice Leadership Academy, Pittsburgh, USA: PA; 2010.

6. Chao S, McQuitty D. Medical, psychological, psychosocial, psycho education evaluations. In: Wilson KB, et al. editors. Case management for the health, human and vocational rehabilitation services. Linn Creek, Aspen, USA; 2018. p. 72-96.

7. Acklin CL. Case management, mental health, and substance abuse. In: Wilson KB, et al. editors. Case management for the health, human, and vocational rehabilitation services. Linn Creek, Aspen, USA; 2018. p. $230-257$.

8. Derr AS. Mental health service use among immigrants in the United States: A systematic review. Psychiatr Serv. 2015;67(3):265-274

9. Substance Abuse and mental health services administration. Mental health services administration, racial/ethnic differences in mental health service use among adults. MD: Substance Abuse and Mental Health Services Administration. Rockville, USA: HHS Publication; 2015.

10. Sue D, Arredondo P, McDavis R. Multicultural counseling competencies and standards: A call to the profession. Journal of Multicultural Counseling and Development. 1992;70(4):477-486. 